\title{
Influence de la Réactivation de la Faille de Kandi sur l'artésianisme dans le Bassin Versant du Fleuve Mono au Sud-ouest du Bassin Sédimentaire Côtier du Bénin (Afrique de l'Ouest)
}

\author{
Luc Adissin Glodji, PhD
}

Département des Sciences de la Terre,

Faculté des Sciences et Techniques, Université d'Abomey-Calavi, Bénin

Abdoukarim Alassane, PhD

Prudence Mahutondji Dossou, PhD Student, MSc

Laboratoire d'Hydrologie Appliquée, Institut National de l'Eau (INE), Université d'Abomey-Calavi, Bénin

André Zogo, Armel Ahossi, PhD Student

Partenariat National de l'Eau, Direction Générale de l'Eau, Bénin

Vinel Gbewezoun, PhD Student

Laboratoire d'Hydrologie Appliquée, Institut National de l'Eau (INE),

Université d'Abomey-Calavi, Bénin

Doi:10.19044/esj.2019.v15n24p346 URL:http://dx.doi.org/10.19044/esj.2019.v15n24p346

\section{Résumé}

La projection des coordonnées géographiques des points d'artésianime de l'aquifère du Crétacé supérieur sur la carte des fractures du Sud-Ouest du bassin sédimentaire côtier du Bénin a révélé le lien étroit entre les forages artésiens et les fracturations. Les forages artésiens sont alignés sur les fractures d'orientation NNE-SSW qui proviennent de la réactivation de la faille de Kandi. L'artésianisme de l'aquifère du Crétacé supérieur qui se traduit par les forages jaillissants et les suintements d'eau serait donc lié à l'effet conjugué de la topographie, de l'agencement des couches géologiques et des failles.

Mots-clés: Fracture, Artésianisme, Aquifère, Crétacé supérieur, Bénin 


\title{
Influence of Kandi Fault Reactivation on Artesianism in the Mono River Watershed at the South-West of Bénin Coastal Sedimentary Basin (West Africa)
}

\author{
Luc Adissin Glodji, PhD
}

Département des Sciences de la Terre,

Faculté des Sciences et Techniques, Université d'Abomey-Calavi, Bénin

Abdoukarim Alassane, PhD

Prudence Mahutondji Dossou, PhD Student, MSc

Laboratoire d'Hydrologie Appliquée, Institut National de l'Eau (INE),

Université d'Abomey-Calavi, Bénin

André Zogo,

Armel Ahossi, PhD Student

Partenariat National de l'Eau, Direction Générale de l'Eau, Bénin

Vinel Gbewezoun, PhD Student

Laboratoire d'Hydrologie Appliquée, Institut National de l'Eau (INE),

Université d'Abomey-Calavi, Bénin

\begin{abstract}
The projection of geographic coordinates of artesianism points of Upper Cretaceous aquifer on the fractures map of southwestern part of the Bénin coastal sedimentary basin revealed a significant relationship between artesian wells and fracturations. The artesian wells are aligned along fractures oriented NNE-SSW derived from the reactivation of Kandi fault. The artesianism of Upper Cretaceous aquifer, resulting in drilling wells and water oozing, would be related to effects of topography and the arrangement of geological layers and faults.
\end{abstract}

Keywords: Fracture, Artesianism, Aquifer, Upper Cretaceous, Benin

\section{Introduction}

L'eau est une ressource indispensable à la survie de l'homme et au développement de ses activités. Au Bénin, les réserves en eau souterraine sont réparties dans les formations géologiques des bassins sédimentaires et dans les altérites et fractures du socle cristallin. Dans la partie méridionale du Bénin s'observe le bassin sédimentaire côtier qui occupe approximativement $10 \%$ 
du territoire et renferme environ $35 \%$ des ressources en eaux souterraines du pays (DGH, 2000).

Le bassin sédimentaire côtier repose en discordance majeure sur le socle cristallin. Il s'est formé au même moment que ses homologues du Golfe de Guinée tel que le bassin de la Côte d'Ivoire (Douzo et al., 2019). Il comporte les formations géologiques du Crétacé supérieur, du Paléogène, du Néogène et du Quaternaire qui sont structurées en huit unités lithostratigraphiques (Breda, 1989; Kaki et al., 2001; Oyédé et al., 2006). Des failles de direction NE-SW à ENE-WSW sont cartographiées dans la partie septentrionale à l'Est et au Centre du bassin. Par contre dans la partie sudouest du bassin, les failles sont d'orientation NNE-SSW (Figure 1) (Breda, 1989). Les failles de la partie sud-ouest du bassin résultent de la réactivation de la faille de Kandi dont la dernière manifestation tectonique remonte au Miocène (Guiraud \& Alidou, 1981; Breda, 1989; Konaté, 1999; Adissin Glodji, 2012). Du point de vue hydrogéologique, il est dénombré quatre niveaux aquifères à l'échelle du bassin sédimentaire côtier. Ce sont, de bas en haut, les aquifères du Crétacé supérieur, de l'Eo-paléocène, du Mio-Pliocène (Continental terminal) et du Quaternaire (Boukari \& Alassane, 2007; Boukari, 2012).

Bien que l'aquifère du Crétacé supérieur soit présente sur l'ensemble du bassin sédimentaire côtier, le phénomène de l'artésianisme de cet aquifère est plus fréquent dans la partie sud-ouest du bassin qu'au Centre et à l'Est. Dans cette partie du bassin, le phénomène de l'artésianisme s'observe en de nombreux points et des forages jadis à motricité humaine deviennent artésiens et d'autres qui étaient éteints commencent subitement à jaillir de l'eau. Ces manifestations suscitent d'énormes interrogations et inquiétudes de la part des autorités politico-administratives et des populations de cette partie du bassin sédimentaire côtier.

Le but de cet article est d'expliquer les causes de la différence de comportement hydrogéologique de l'aquifère du Crétacé supérieur dans la partie sud-ouest par rapport au Centre et l'Est du bassin sédimentaire côtier. Pour ce faire, les caractéristiques hydrogéologiques de cet aquifère supérieur sont étudiées à l'échelle du bassin versant du Mono qui couvre la zone d'étude (Figure 2). Le bassin versant du fleuve Mono est localisé sur les roches du bassin sédimentaire côtier dans sa partie méridionale et les roches du socle cristallin dans sa partie septentrionale (PNE-Bénin, 2015, 2016). Au Nord-Est du bassin versant du Mono, s'observent des blastomylonites et mylonites liées à la zone de failles de Kandi (Figure 2). 


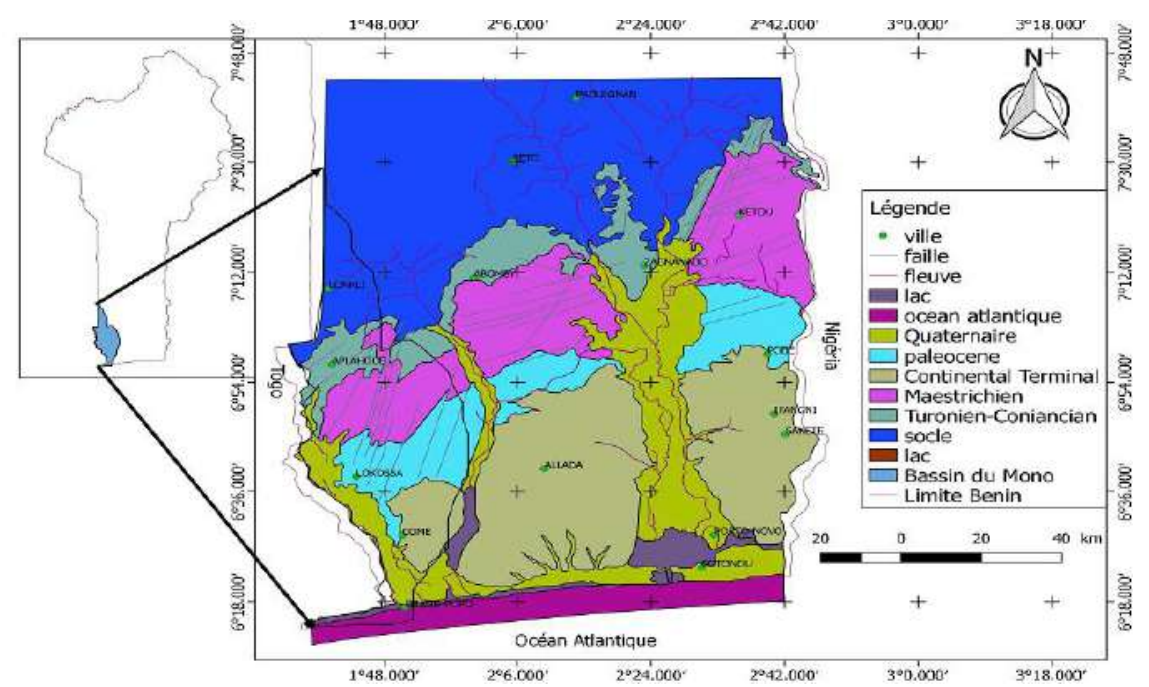

Figure 1. Carte géomorphologique du bassin sédimentaire côtier du Bénin montrant la localisation du bassin versant du Mono (Slansky, 1962 modifié d'après Breda, 1989)

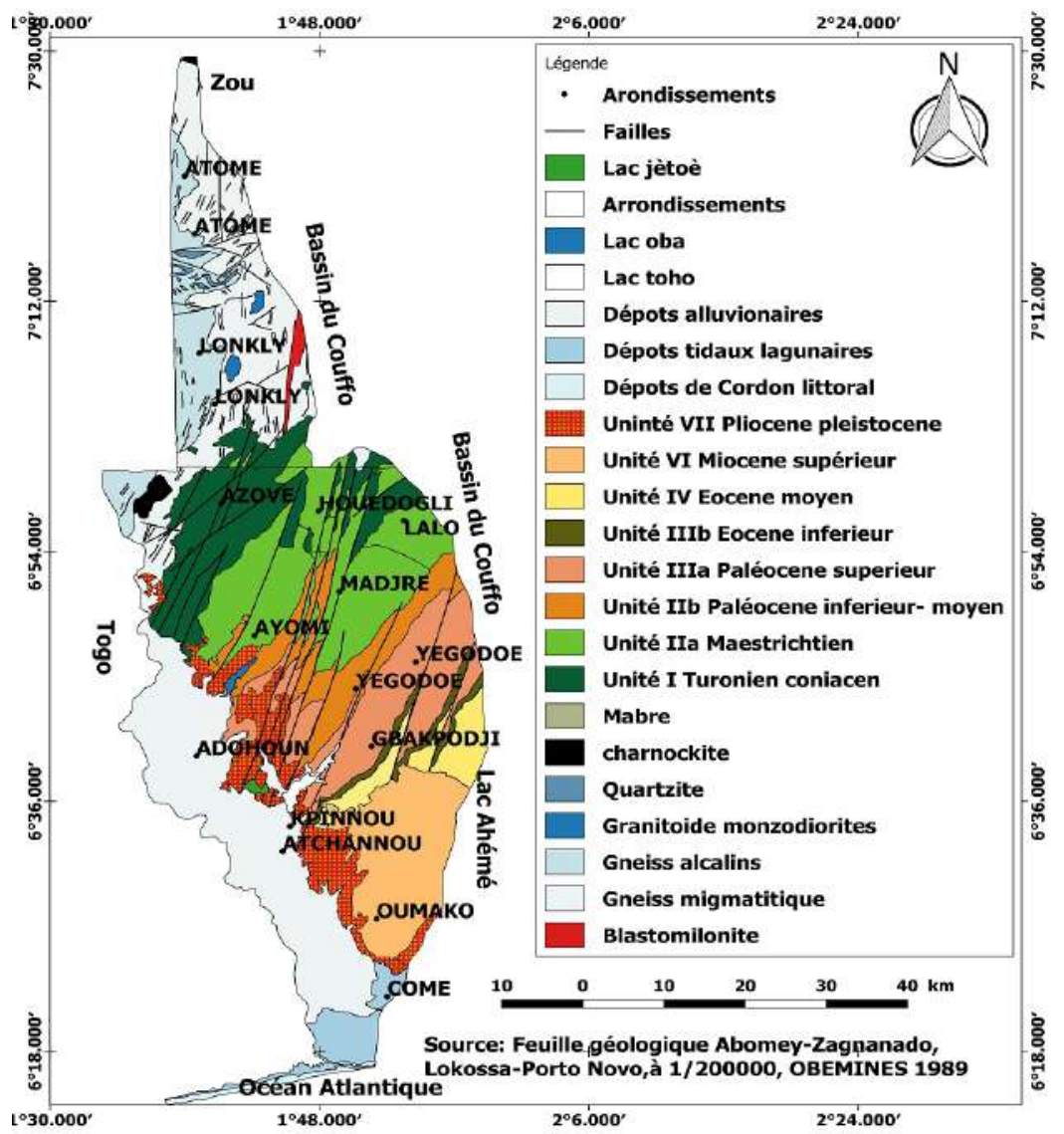

Figure 2. Carte géologique du bassin versant du Mono au Bénin (extraite des feuilles géologiques à 1/200000 Lokossa-Porto Novo et Abomey-Zangnanado (Breda, 1989)) 


\section{Matériel et méthodes}

La méthodologie de recherche adoptée pour cette étude comporte essentiellement la collecte des données lithostratigraphiques des forages ; les travaux de terrain pour la cartographie des fractures et la constitution de la base des coordonnées des forages artésiens du bassin versant du Mono et enfin l'analyse et le traitement des données.

\subsection{Collecte des données}

Les données collectées concernent les logs des forages réalisés dans les formations géologiques du Crétacé supérieur. Ces données ont été collectées auprès de la Direction Générale de l'Eau (DG-Eau) et l'Office Béninois de Recherches Géologiques et Minières (OBRGM) du Bénin.

\subsection{Travaux de terrain}

Les travaux de terrain ont été menés dans l'objectif de faire l'inventaire des forages artésiens de la zone d'étude et de prendre les coordonnées géographiques de ces points artésiens au moyen d'un GPS. Ceci a permis de mettre à jour la base des coordonnées géographiques des forages artésiens du bassin du fleuve Mono. Aussi, des fractures ont été cartographiées dans le bassin versant du Mono sur le terrain.

\subsection{Analyse et traitement des données}

Les données obtenues ont permis de réaliser les cartes et coupes hydrogéologiques à l'aide des logiciels QGIS 2.18. Pour les coupes hydrogéologiques, nous avons utilisé les cartes topographiques à l'échelle de $1 / 200000^{\mathrm{ème}}$ (feuille Lomé et Abomey) et les logs stratigraphiques des forages situés dans la zone ou affleure le Crétacé supérieur dans le bassin versant du Mono. Cette démarche consiste à projeter les forages sélectionnés sur la carte topographique, exécuter les profils topographiques le long des traits de coupes, positionner les logs des forages à proximité des traits de coupe de même que les niveaux statiques sur le profil réalisé. Ensuite nous avons ajusté les niveaux statiques et corréler les niveaux piézométriques des horizons captés. Nous avons effectué les corrélations lithostratigraphiques entre les différents logs. Enfin les coupes hydrogéologiques ont été numérisées dans le logiciel QGIS 2.18 après les avoir scanné. Pour mieux décrire la productivité des aquifère captés de la zone d'étude, trois coupes hydrogéologiques ont été réalisées suivant les traits de coupe AA' orientés NNW-SSE et BB'et CC' de direction W-E (Figure 3).

La rosace directionnelle des fractures a été réalisée avec le logiciel Geo orient 3.2. Une corrélation a été effectuée entre les failles et les points de forage artésiens du bassin versant du Mono. Pour ce faire, les coordonnées 
géographiques des points de forage artésien sont projetées sur la carte des fracturations afin de déterminer leur répartition spatiale par rapport aux failles.

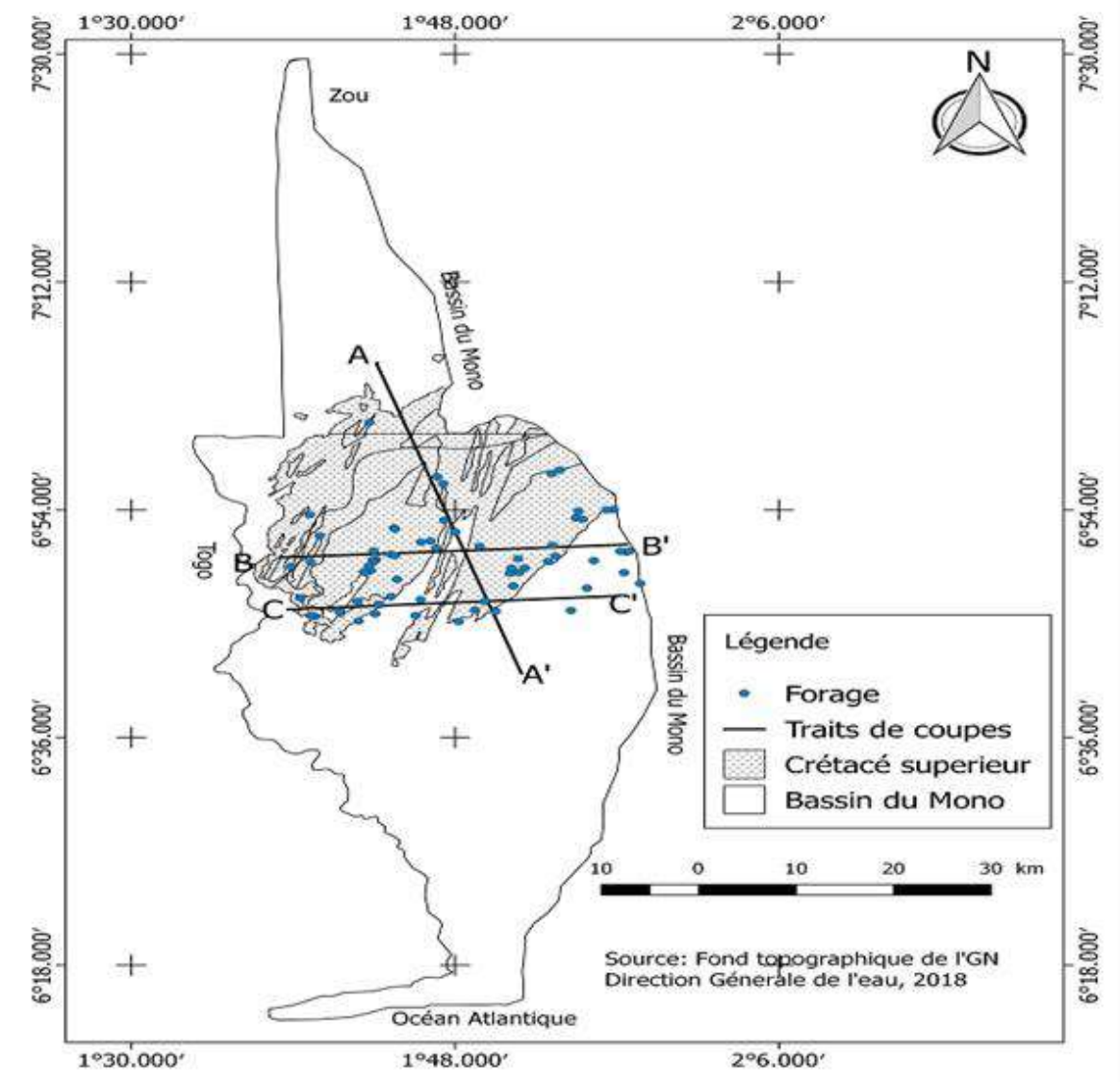

Figure 3. Carte du bassin du Mono montrant les traits de coupes réalisés

\section{Résultats}

\subsection{Cartographie des points d'artésianisme du bassin versant du fleuve Mono}

Nous avons dénombré 86 points d'artésianisme dans le bassin versant du Mono (Figure 5). Ces points artésiens sont soit des puits à grand diamètre devenus artésiens par la suite (Figure $4 \mathrm{a}, \mathrm{b}$ ) ou soit des forages équipés de pompe à motricité humaine (FPM) éteints et qui commencent subitement à jaillir de l'eau (Figure 4c, d, e, f). Certains sites où l'eau jaillit ou suinte se présentent actuellement sous forme de bas-fonds dont quelques-uns sont en cours d'aménagement à usage agricole. L'eau suinte actuellement dans une salle de classe d'une école primaire construite à l'emplacement d'un ancien forage éteint dont les éléments de la superstructure et la pompe avaient été enlevés (Figure 4e, f). Cette eau est utilisée pour les activités de transformations alimentaires dans la localité. 

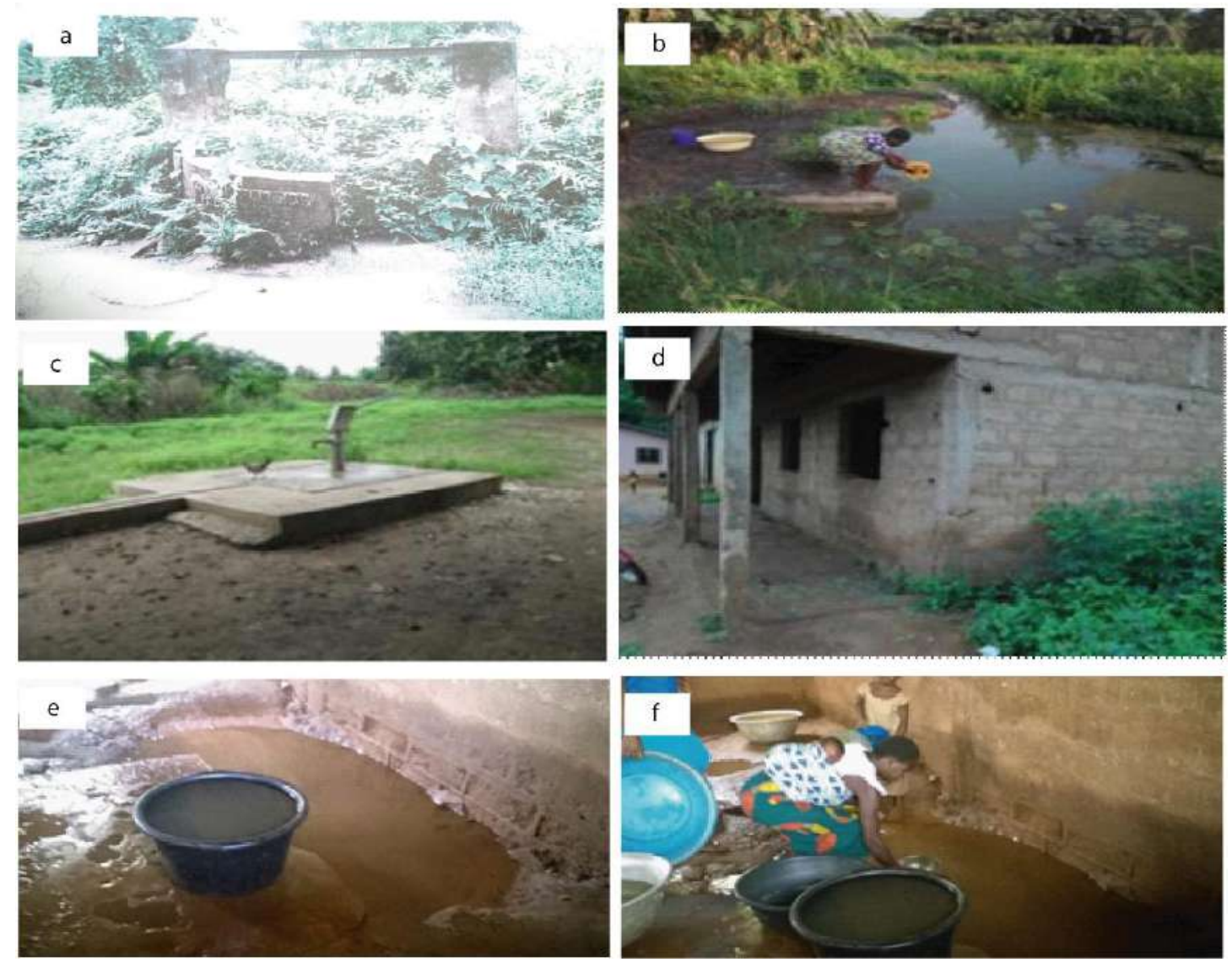

Figure 4. Photographies montrant l'aspect de quelques points artésiens dans le bassin versant du fleuve Mono. (a) aspect d'un puits avec sa margelle à la réalisation, (b) et devenu artésien après à Tannou-Gola (Commune de Toviklin), (c) FPM avant la construction de l'école maternelle à l'EPP de Dékandji, (d) construction de l'école maternelle à l'EPP de Dékandji, (e) FPM devenu artésien à l'EPP de Dékandji, (f) exploitation de l'eau à l'EPP de Dékandji, arrondissement de Tota (Commune de Dogbo). 


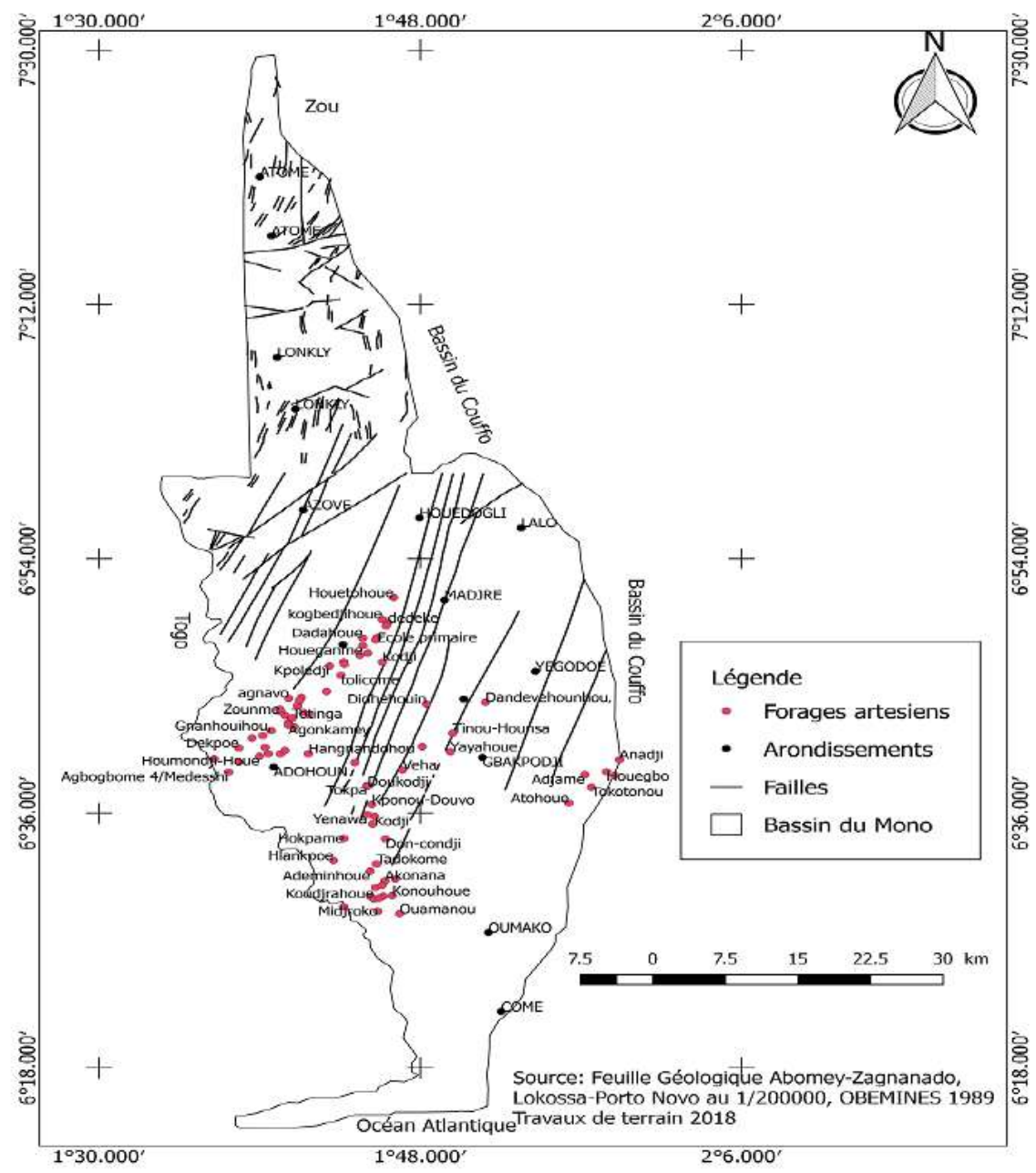

Figure 5. Carte des fractures du bassin versant du Mono montrant la localisation points artésiens.

\subsection{Structure et géométrie de l'aquifère du Crétacé supérieur}

Les figures 6,7 et 8 représentent les coupes hydrogéologiques réalisées respectivement suivant les traits de coupe AA', BB' et CC'.

La coupe hydrogéologique de la Figure 6 met en évidence 4 formations hydrogéologiques qui diffèrent légèrement dans la partie nord et dans la partie sud. Au Nord, s'observent du haut vers le bas, les couches sableuse et argilosableuse qui reposent sur les altérites (schistes) du socle cristallin ou par l'intermédiaire des grès. Dans la partie sud, une couche latéritique parfois cuirassée s'observe à la surface. Sous cette couche, s'observent successivement les couches de sable argileux et d'argile à passées calcareuses qui reposent sur un niveau sableux. Les horizons captés par les forages sont les couches de sable et de grès. Cette géométrie laisse supposer que 
l'artésianisme n'est pas possible dans la partie nord du bassin versant du Mono $\mathrm{du}$ fait de l'inexistence d'une couche argileuse d'épaisseur importante. Par contre dans la partie sud, l'épaisseur importante de la couche argileuse imperméable au-dessus du niveau sableux laisse suggérer que l'artésianisme est possible.

La coupe hydrogéologique réalisée le long du trait de coupe BB' (Figure 7) montre du haut vers le bas, trois (3) couches. Une couche latéritique repose sur une couche argileuse et cette dernière située au-dessus sur une couche sableuse qui renferme localement des lentilles argilo-sableuses ou sablo-argileuses d'épaisseur comprise entre 7 et $10 \mathrm{~m}$. L'aquifère ici est le niveau sableux du Crétacé. L'épaisseur importante de la couche argileuse au toit de l'aquifère sableux laisse supposer que l'artésianisme est possible le long de cette coupe à partir de la localité de Démanhouéhoué vers l'Est (Figure 7).

La Figure 8 représente la coupe hydrogéologique W-E suivant le trait CC'. Elle révèle cinq (05) types de couches lithologiques à savoir la couche latéritique, semi imperméable, la couche argileuse très épaisse qui est une formation hydrogéologique imperméable, la couche sablo- argileuse, semi imperméable et argilo-sableuse sous forme de lentille, et enfin la couche sableuse qui représente le niveau aquifère. La couche argileuse est présente dans toutes les localités et a une épaisseur comprise entre 30 et 35 mètres. Les crépines sont placées dans la couche sableuse qui est l'aquifère dont l'épaisseur est variable de 15 à 25 mètres. Au vu du volume de cette couche argileuse présente dans toutes les localités et qui représente le toit de l'aquifère sableux, l'artésianisme serait possible sur toute la superficie de la zone.

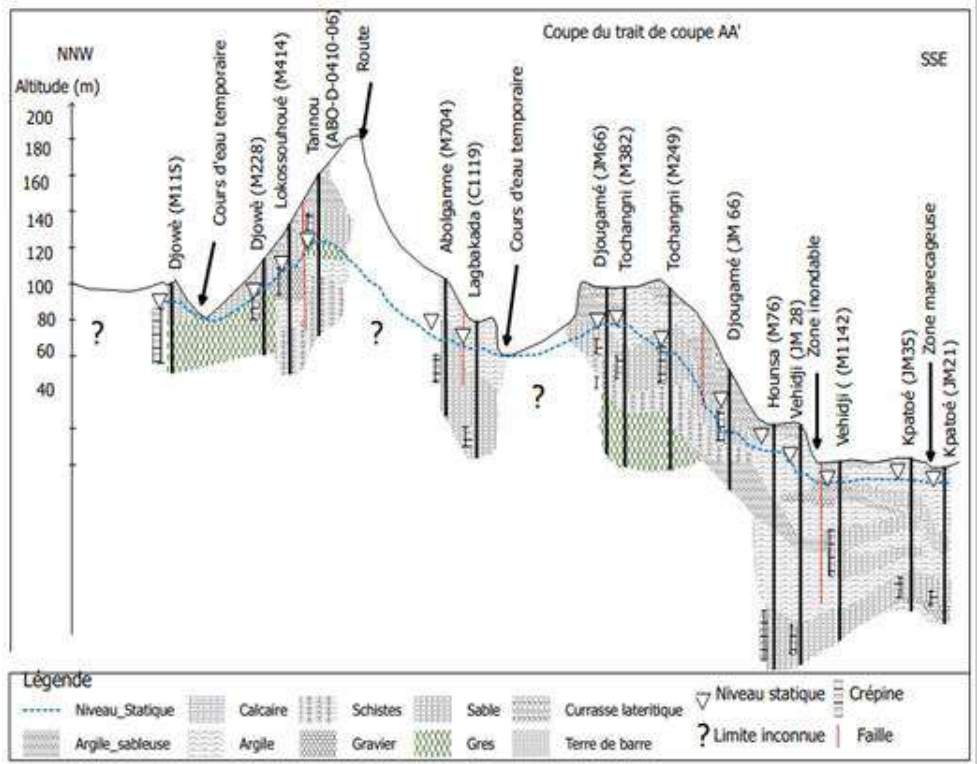

Figure 6. Coupe hydrogéologique NNW-SSE suivant le trait de coupe AA' 


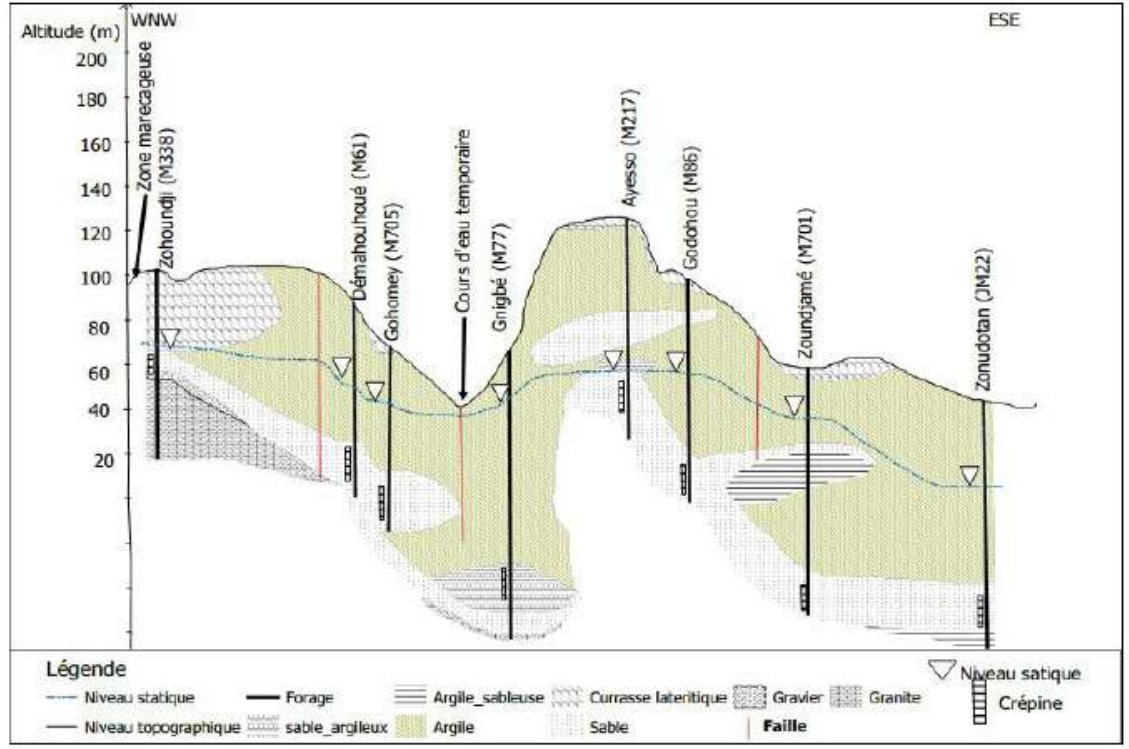

Figure 7. Coupe hydrogéologique W-E suivant le trait de coupe BB'

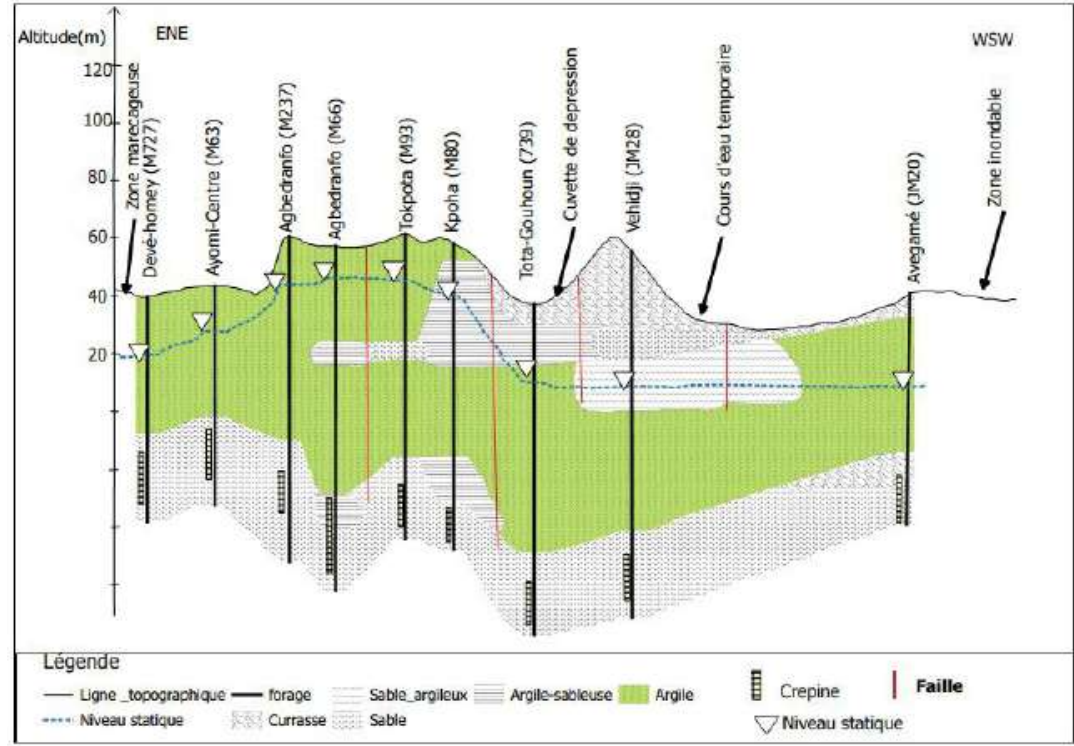

Figure 8. Coupe hydrogéologique W-E suivant le trait de coupe CC'

\subsection{Analyse des fracturations dans le bassin versant du Mono}

La Figure 5 représente la carte des fracturations du bassin versant du Mono. La Figure 9 représente la rosace des directions des fracturations du bassin. Les directions majeures des fracturations dans le bassin versant du Mono varient du N-S à NNE- SSW (Figure 9). Environ $80 \%$ des fractures présentent une orientation comprise dans cet intervalle. La direction résultante est $\mathrm{N} 16^{\circ}$. De plus des fractures de direction NE-SW et NNW-SSE sont 
présentes mais en faible pourcentage (6\% pour chaque direction) surtout dans le socle cristallin de la partie nord du bassin versant du Mono.

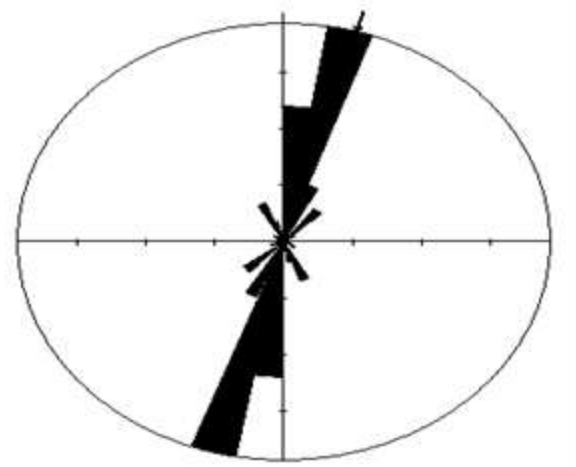

Figure 9. Rosace des directions de fracturations du bassin du Mono

\subsection{Lien entre les fractures et les forages artésiens}

La Figure 5 montre la position des forages artésiens par rapport aux fractures dans le bassin versant du fleuve Mono. De l'observation de la Figure 5, on s'aperçoit que la distribution spatiale des forages artésiens dans le bassin du Mono n'est pas aléatoire. La majorité de ces forages artésiens est alignée le long des fractures comme dans les localités de Kodji, Adohoun, Tokpa, Veha, Diahehouin, Yénama, Kodji, Tinou-hounsa, Yayahoué et Dankodji. La distribution spatiale de ces forages suit la même orientation que celle des fracturations dans le bassin versant. Le premier exemple d'orientation d'un ensemble des forages est celui des forages qui quittent la partie sud-ouest du bassin (Agbogbomé-Houmondjihoué-Dekpoé-Tetinga), passant par Toliomé-Kodji-Ecole primaire-Dedeke et Houétohoué vers le Nord-Est. L'orientation de cet ensemble de forages coincide avec la direction des fracturations d'orientation NNE-SSW décrite. Un second ensemble d'orientation des forages est celui qui quitte le Sud-Ouest du bassin (Koudrahoué, Akonana, Tadokomé), passant par le milieu du bassin (Doucondji, Yayahoué) vers le Nord-Est du bassin (Tinou-hounsa et Dandevehounhoué). L'ensemble de ces forages suit la même direction que celle des fracturations et alignés sur une zone de fractures majeures. Ces résultats montrent la forte corrélation entre l'alignement des forages artésiens et les fractures dans le bassin versant du fleuve Mono.

\section{Discussion}

La partie sud-ouest du bassin sédimentaire côtier du Bénin se caractérise du point de vue hydrogéologique par un phénomène d'artésianisme qui se traduit par des forages jaillissants et le suintement d'eau. Ce phénomène est particulièrement observé à l'Ouest du bassin. Les études menées dans la partie sud-ouest du bassin sédimentaire sur le bassin versant du fleuve Mono 
permettent de mettre en exergue les facteurs responsables de ce phénomène hydrogéologique.

Dans le bassin versant du Mono, l'aquifère concerné par ce phénomène est celui des sables du Crétacé supérieur reposant en discordance majeure sur le socle cristallin et recouvert par une couche argileuse d'épaisseur comprise entre 15 et $85 \mathrm{~m}$. Cette couche argileuse tend à compresser l'aquifère sableux et le rend ainsi en charge. Cette succession stratigraphique semble être uniforme sur l'ensemble de la partie méridionale du bassin côtier (Slansky, 1962; Breda, 1989) mais le comportement hydrogéologique de l'aquifère du Crétacé supérieur est différent à l'Ouest que celui observé au Centre et l'Est du bassin.

Selon Kresic and Stevanovic (2010) et Sabrina (2012), la topographie et la structure géologique jouent un rôle important sur l'artésianisme. La structure géologique exprime non seulement la géométrie des couches mais aussi les déformations essentiellement discontinues qu'elles ont subies. Les zones de déformations discontinues sont des zones de faiblesse structurale qui peuvent servir de drain pour l'eau contenue dans un aquifère en charge en favorisant sa remontée artésienne. Dans un tel contexte, les forages positionnés dans les aquifères en charge et intensément fracturés seront plus facilement jaillissants et l'eau dans sa remontée peut aussi s'infiltrer le long des fractures et suinter en de nombreux endroits aux environs des points de forage. Ceci expliquerait la fréquence élevée de l'artésianisme au Sud-Ouest du bassin sédimentaire côtier.

Cette abondante fracturation dans la partie sud-ouest est liée à la réactivation de la faille de Kandi. En effet, la faille de Kandi est un accident tectonique régional qui s'étend depuis le Hoggar en Algérie à travers le Niger, le Bénin et qui se prolonge au Sud du Brésil (Trompette, 1994; Adissin Glodji, 2012; Adissin Glodji et al., 2014). La partie sud de la faille de Kandi sur le territoire du Bénin est située dans le bassin versant du fleuve Mono. Dans la partie septentrionale du bassin versant du Mono formée du socle cristallin, les manifestations de cette faille sont matérialisées par une bande de largeur kilométrique orientée N-S formée de cataclasites (roches broyées). Dans la partie sud du bassin versant du Mono, cette faille plonge sous les sédiments du bassin sédimentaire côtier. L'activité tectonique initiale de la faille de Kandi remonte à la fin du Précambrien (Adissin Glodji, 2012). L'histoire structurale du bassin versant du Mono est étroitement liée à l'histoire polyphasée de la faille de Kandi. Cet accident a été réactivé pendant le Paléozoïque. Au cours du Crétacé, cette faille a joué en faille décrochante orientée N-S (Breda, 1989). Au cours de l'Oligocène supérieur au Miocène supérieur, et pendant l'intervalle de temps compris entre les phases d'érosion et / ou de non dépôt, cette faille a rejoué en donnant lieu à trois systèmes de failles orientées N-S à NNE-SSW pour les failles majeures (Exemple de la 
faille de Lokossa) et NE-SW et ESE-WNW pour les secondaires (Breda, 1989).

La confrontation de la carte des fractures du bassin versant du Mono avec les coordonnées des points de forages artésiens conforte le lien entre les forages artésiens et les fracturations. Les forages se développent le long des fracturations d'orientation NNE-SSW qui résultent de la réactivation de la faille de Kandi. D’après ces différents résultats, l'artésianisme de l'aquifère du Crétacé supérieur qui se traduit par les forages artésiens et les suintements d'eau est lié à l'effet conjugué de la topographie, de la disposition des couches géologiques et des structures tectoniques.

\section{Conclusion}

Cette étude a permis à l'aide de la géologie structurale, l'hydrogéologie et des données des logs disponibles sur les forages du bassin versant du fleuve Mono d'identifier les causes possibles de la fréquence élevée de l'artésianisme dans la partie occidentale du bassin sédimentaire côtier. Les zones favorables à l'artésianisme se situent dans la partie sud-ouest de l'aquifère du Crétacé supérieur le long des fractures d'orientation NNE-SSW. Par contre au Nord, cet aquifère qui constitue le principal réservoir des forages artésiens est libre. Pour minimiser les risques de pollution de cet aquifère, nous recommandons que ces zones soient cartographiées et protégées. Par ailleurs, pour éviter le gaspillage des eaux des forages artésiens, les initiatives de gestion intégrée des ressources en eau (GIRE) doivent être mises en place autour de ces forages artésiens. A la longue, la variation temporelle des niveaux de la nappe captive du Crétacé supérieur sera modélisée dans le bassin sédimentaire côtier du Bénin.

\section{References:}

1. Adissin Glodji, L. (2012). La zone de cisaillement de Kandi et le magmatisme associé dans la region de Savalou-Dassa (Bénin) : Etude structurale, pétrographique et géochronologique. Thèse de doctorat, Université jean Monnet Saint Etienne et Unversité Abomey-Calavi, $277 \mathrm{p}$.

2. Adissin Glodji, L., Bascou, J., Yessoufou, S., Ménot, R.P., \& Villaros, A. (2014). Relationships between deformation and magmatism in the Pan-African Kandi Shear Zone: Microstructural and AMS study studies of Ediacaran granitoids intrusions in central Bénin (West Africa). Journal of African Earth Sciences, 97, 143-160.

3. Boukari, M. (2012). Réactualisation des connaissances hydrogéologiques relatives, au bassin sédimentaire côtier du Benin. Rapport, Direction de l'Hydraulique, Cotonou, Bénin, 137p.

4. Boukari, M. \& Alassane, A. (2007). Les ressources en eau souterraine 
du bassin sédimentaire côtier de la République du Bénin. Africa Géoscience review, 14 (3), 283-301

5. Breda (1989). Notice explicative de la carte géologique à 1/200000, Feuille Pira-Savè, Abomey-Zagnanado et Lokossa-Porto Novo, mémoire $\mathrm{n}^{\circ} 3$, 1ere édition, $77 \mathrm{p}$.

6. DGH (2000). Politique nationale de gestion des ouvrages hydrauliques. Rapport, Cotonou, 39 p.

7. Douzo, J.W.D. et al. (2019). Lithostratigraphical and Sedimentological Characterization of Deposits in the Fresco and Grand Lahou (Southwest Cote d'Ivoire): Correlation Attempt and Paleoenvironments. European Scientific Journal, 15 (15), 403-424

8. Guiraud, R. \& Alidou, S. (1981). La faille de Kandi (Bénin), témoin du rejeu fini-crétacé d'un accident majeur à l'échelle de la plaque africaine. Comptes Rendus de l'Académie des Sciences de Paris, 293, 779-782.

9. Kaki, C., Oyédé, L.M., \& Yessoufou, S. (2001). Dynamique sédimentaire et environnement côtier béninois à l'Est de l'embouchure du fleuve Mono. Rech. Sci. Univ. Lomé (Togo), 5 (2), 247-261

10. Konaté, M. (1996). Evolution tectono-sédimentaire du bassin paléozoïque de Kandi (Nord Bénin, Sud Niger). Un témoin de l'extension post-orogénique de la chaîne panafricaine. Thèse de doctorat, Université de Dijon, France, 489 p.

11. Kresic, N. \& Stevanovic, Z. (2010). Hydrology of springs: engineering and sustainability. Acta Carsologica, 39 (2), 573 p.

12. Oyédé, L.M., Tossou, M.G., Kaki, C., Laibi, R.A. \& Lang, J. (2006). Phénomènes enregistrables, milieu enregistreur et messages enregistrés: application aux séquences biosédimentaires du quaternaire récent dans le géosystème margino-littoral béninois (Afrique de l'Ouest). Africa Géoscience Review, 13 (3), 395-408.

13. PNE-Bénin (2015). Amélioration de la connaissance sur le phénomène de l'artésianisme dans le Mono et Couffo, Rapport de mission, 8 p.

14. PNE-Bénin (2016). Documentation des pratiques et problématiques de gestion durable des ressources en eau dans la portion béninoise du bassin du fleuve Mono, rapport final, 132p.

15. Sabrina, C. (2012). Hydrogéochimie des sources associées aux eskers de l'Abitibi, Québec, école polytechnique de Montréal. Mémoire de maitrise es-science appliquée, 58p.

16. Slansky, M. (1962). Contribution à l'étude géologique du bassin sédimentaire côtier du Dahomey et du Togo. Mém. N¹1 du B.R.G.M.

17. Trompette, R. (1994). Geology of Western Gondwana (2000-500 Ma). Pan-African-Brasiliano Aggregation of South America and Africa. A.

A. Balkema, Rotterdam 350 p. 